\title{
Optimization of Direct Power Control of Three-Phase Active Rectifiers by using Multiple Switching Tables
}

\author{
J.G. Norniella, J.M. Cano, G.A. Orcajo, C.H. Rojas, J.F. Pedrayes, M.F. Cabanas, M.G. Melero \\ University of Oviedo \\ Electrical Engineering Dept. \\ Campus de Viesques - 33204 Gijón (Spain) \\ Fax:+34 985182453, e-mail: jgnorniella@uniovi.es,jmcano@uniovi.es, gonzalo@uniovi.es, chrojas@uniovi.es, \\ pedrayesjoaquin@uniovi.es, manes@uniovi.es, melero@uniovi.es
}

\begin{abstract}
One of the best known control methods for threephase active rectifiers is the so-called Direct Power Control (DPC). Control algorithm in DPC is based on the regulation of instantaneous active and reactive powers and incorporates a switching table where the successive optimal configurations of the rectifier are stored. Traditional DPC models use only one switching table within their control systems. In this study, a new DPC method is presented, based on the combination of two different switching tables. By using Simulink simulations, it is demonstrated that better dynamics and lower switching stress are achieved in the rectifier thanks to the use of the proposed method, in comparison to the simple switching table models.
\end{abstract}

\section{Key words}

Direct power control, instantaneous active and reactive powers, switching table, three-phase active rectifier, power quality, $\mathrm{p}-\mathrm{q}$ theory.

\section{Introduction}

\section{A. Voltage source three-phase rectifiers and DPC}

Main problems derived from the use of traditional diode/thyristor-based AC/DC three-phase rectifiers are the harmonic pollution induced and the associated low power factor. So as to face these drawbacks, rectifiers made up of controllable power switches are developed. In this way, improvement in power factor and decrease in harmonic components can be achieved by active control of converter input currents.

Some of these rectifier topologies (Boost, Viena) are more appropriate for applications where the energy is only transmitted from source to load. However, there are other situations in which energy flux must be reverted; in those cases, the so-called active (front end) rectifiers (AFE's) represent a proper solution. There are two main types of AFE's: current source rectifiers (CSR's), which are required to deliver a constant output current, and voltage source rectifiers (VSR's) (Fig. 1), which are supposed to achieve a constant output voltage.

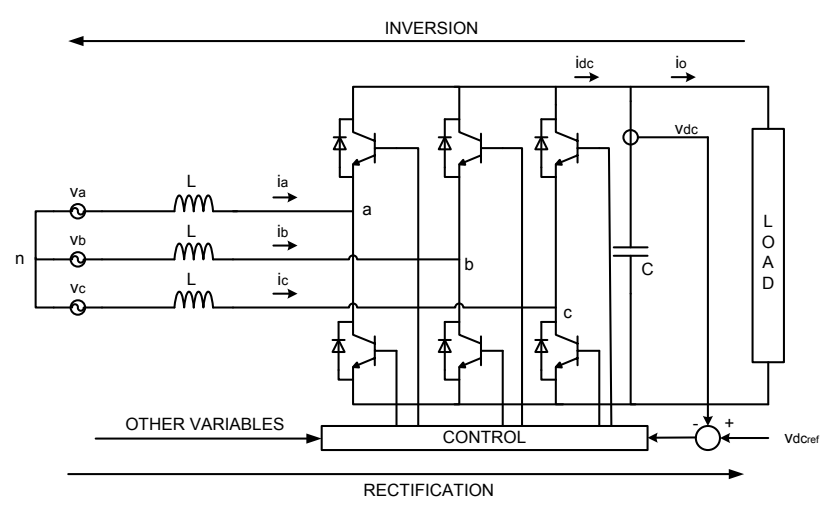

Fig. 1. Typical topology of a voltage source three-phase active rectifier

Use of AFE's requires the incorporation of a control system in order to achieve their proper performance. Traditional Voltage-oriented control (VOC) strategy [1] guarantees a proper static and dynamic performance via implementation of inner current loops. However, its operation strongly depends on the accuracy of current components separation, the proper design of controllers and the correct adjustment of their parameters. In connection with these problems, Direct Torque Control (DTC) methods for electrical machines [2] avoid the need of current regulators and provide a direct control of torque and flux. Based on DTC's operation principles, Direct Power Control (DPC) methods for three-phase active rectifiers were developed [3],[4]. In this paper, an improvement in DPC for VSR's in order to achieve better dynamics and lower switching stress is presented.

\section{B. Commutation state vectors}

As can be seen in Fig. 1, three-phase active rectifiers are made up of three half-bridges, each one of them being constituted by two switches. Assuming that one (and only one) of the switches in each branch must always be closed when proper operation of the rectifier is achieved, 
only eight possible converter configurations are possible [5]. Commutation states of the three upper switches (1 if closed, 0 if open) identify the general commutation state of the rectifier, those being the components $\left(\mathrm{S}_{\mathrm{a}} \mathrm{S}_{\mathrm{b}} \mathrm{S}_{\mathrm{c}}\right)$ of the so-called (converter) commutation state vectors (V0V7), which are shown in Table I.

TABLE I. - Commutation state vectors

\begin{tabular}{|c|c|}
\hline Vector & Commutation state $\left(\mathrm{S}_{\mathrm{a}} \mathrm{S}_{\mathrm{b}} \mathrm{S}_{\mathrm{c}}\right)$ \\
\hline $\mathrm{V} 0$ & $(000)$ \\
\hline $\mathrm{V} 1$ & $(100)$ \\
\hline $\mathrm{V} 2$ & $(110)$ \\
\hline $\mathrm{V} 3$ & $(010)$ \\
\hline $\mathrm{V} 4$ & $(011)$ \\
\hline $\mathrm{V} 5$ & $(001)$ \\
\hline $\mathrm{V} 6$ & $(101)$ \\
\hline $\mathrm{V} 7$ & $(111)$ \\
\hline
\end{tabular}

\section{Instantaneous Active and Reactive Power: p-q Theory}

\section{A. Basis of p-q Theory: Clarke Transformation}

Operation of DPC methods is based on the control of instantaneous active and reactive powers, which are defined by the so-called Instantaneous Reactive Power or p-q Theory [6]. This Theory uses Clarke Transformation [6] in order to express phase magnitudes of a three-wire active three-phase rectifier in orthogonal axes $\alpha-\beta$, being those previously placed in a-b-c axes, as seen in Fig. 2 and (1) (analogous equations would be obtained in the case of currents).

$$
\left(\begin{array}{l}
v_{\alpha} \\
v_{\beta}
\end{array}\right)=\sqrt{\frac{2}{3}}\left(\begin{array}{ccc}
1 & -\frac{1}{2} & -\frac{1}{2} \\
0 & \frac{\sqrt{3}}{2} & -\frac{\sqrt{3}}{2}
\end{array}\right)\left(\begin{array}{l}
v_{a} \\
v_{b} \\
v_{c}
\end{array}\right)
$$

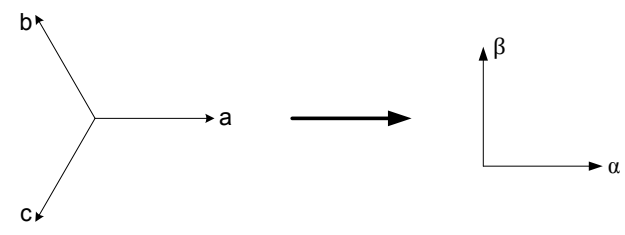

Fig. 2. Transformation from $a-b-c$ to $\alpha-\beta$ axes

The $\sqrt{\frac{2}{3}}$ factor in (1) is essential for power magnitudes to keep their value after the transformation.

\section{B. Vectorial equations in stationary $\alpha-\beta$ axes}

It is possible to define a so-called instantaneous voltage (current) space vector from its $\alpha-\beta$ components, as shown in (2).

$$
\underline{v}=v_{\alpha}+j v_{\beta}
$$

In connection with this, it can be demonstrated that, in the case of balanced sinusoidal three-phase systems, the voltage (current) space vector has constant amplitude and rotates at an angular velocity of $\omega$, being $\omega$ the pulsation of the sinusoidal signals.

\section{Vectorial equations in rotary $d-q$ axes}

It is also possible to define a rotary reference system d-q [7], which rotates, at an angular velocity of $\omega$, in the same direction as the voltage space vector does. Relation between $\alpha-\beta$ and $\mathrm{d}-\mathrm{q}$ systems and decomposition of vector $\underline{v}$ into both pairs of axes are shown in Fig. 3.

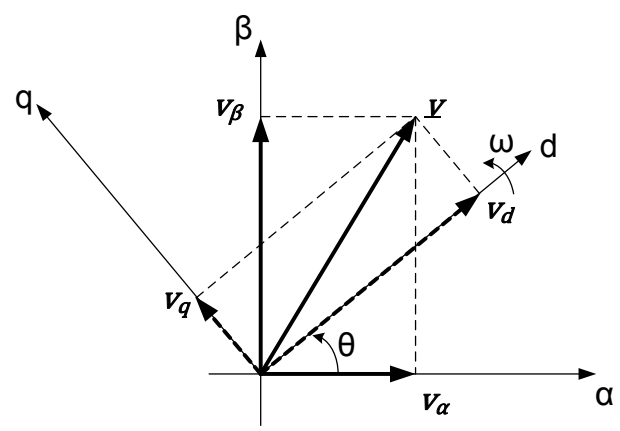

Fig. 3. Relation between $\alpha-\beta$ and $d-q$ axes

Relations between the expressions of vector $\underline{v}$ in $\alpha-\beta$ and d-q systems can be easily obtained from Fig. 3, and are shown in (3).

$$
\begin{aligned}
& v_{d}=v_{\alpha} \cos (\theta)+v_{\beta} \sin (\theta) \\
& v_{q}=-v_{\alpha} \sin (\theta)+v_{\beta} \cos (\theta)
\end{aligned}
$$

\section{Instantaneous powers in $p-q$ Theory}

As Clarke Transformation keeps power values constant after its application, three-phase instantaneous power in a three-wire three-phase active rectifier can be obtained through both of the expressions in (4) [6].

$$
p_{3 \phi}=v_{a} i_{a}+v_{b} i_{b}+v_{c} i_{c}=v_{\alpha} i_{\alpha}+v_{\beta} i_{\beta}
$$

In connection with this, two different instantaneous powers are defined for these electrical systems in $\mathrm{p}-\mathrm{q}$ Theory: instantaneous active $(p)$ and reactive $(q)$ powers [6]. The possibility of calculating them from $\alpha-\beta$ magnitudes, as seen in (5), is the main p-q Theory feature that DPC strategies take advantage of.

$$
\left(\begin{array}{l}
p \\
q
\end{array}\right)=\left(\begin{array}{cc}
v_{\alpha} & v_{\beta} \\
v_{\beta} & -v_{\alpha}
\end{array}\right)\left(\begin{array}{l}
i_{\alpha} \\
i_{\beta}
\end{array}\right)
$$

\section{Direct Power Control}

As can be seen in Fig. 4, DPC is based on the use of control loops for $p$ and $q$ that allow the switches in the rectifier to acquire their optimal configuration, at all times, in order to behave in a specific manner. Apart from this, DPC does not need any source voltage sensors, as it incorporates a method for estimating them from Clarke equations and p-q Theory. These estimations can 
be observed in (6), (7) and (8) [3] (as three-wire system is being considered, $i_{c}$ can be obtained as a function of $i_{a}$ and $i_{b}$ ).

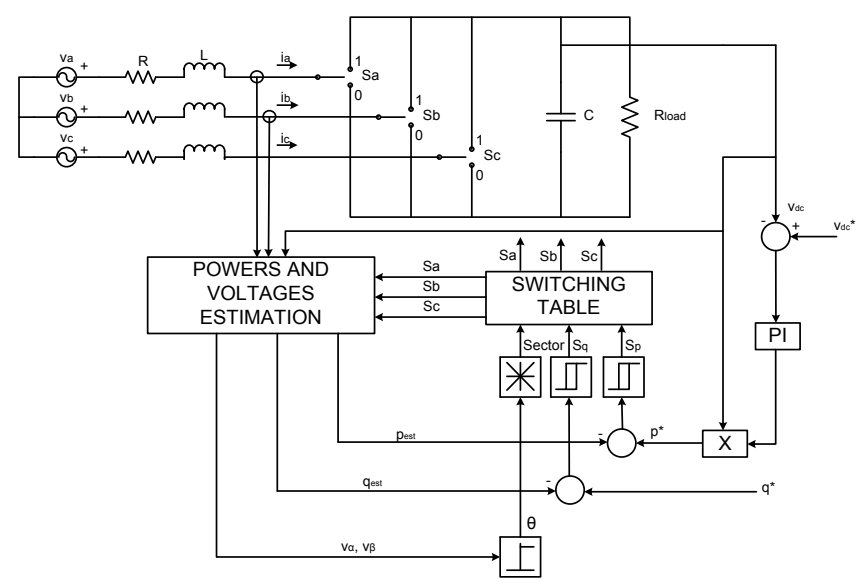

Fig. 4. Basic diagram of DPC control method

$$
\begin{aligned}
& \left(\begin{array}{l}
i_{\alpha} \\
i_{\beta}
\end{array}\right)=\sqrt{\frac{2}{3}}\left(\begin{array}{ccc}
1 & -\frac{1}{2} & -\frac{1}{2} \\
0 & \frac{\sqrt{3}}{2} & -\frac{\sqrt{3}}{2}
\end{array}\right)\left(\begin{array}{l}
i_{a} \\
i_{b} \\
i_{c}
\end{array}\right) \\
& \left(\begin{array}{l}
v_{\alpha_{e s t}} \\
v_{\beta_{e s t}}
\end{array}\right)=\frac{1}{i_{\alpha}^{2}+i_{\beta}^{2}}\left(\begin{array}{cc}
i_{\alpha} & -i_{\beta} \\
i_{\beta} & i_{\alpha}
\end{array}\right)\left(\begin{array}{l}
p_{e s t} \\
q_{e s t}
\end{array}\right) \\
& \left(\begin{array}{l}
v_{a_{e s t}} \\
v_{b_{e s t}} \\
v_{c_{e s t}}
\end{array}\right)=\sqrt{\frac{2}{3}}\left(\begin{array}{cc}
1 & 0 \\
-\frac{1}{2} & \frac{\sqrt{3}}{2} \\
-\frac{1}{2} & -\frac{\sqrt{3}}{2}
\end{array}\right)\left(\begin{array}{l}
v_{\alpha_{e s t}} \\
v_{\beta_{e s t}}
\end{array}\right)
\end{aligned}
$$

As can be noticed in (7), the estimation of instantaneous active and reactive powers takes part, in turn, in the estimation of source voltages. In connection with this, instantaneous powers can be estimated as shown in (9) and (10).

$$
\begin{aligned}
& p_{e s t}=v_{a} i_{a}+v_{b} i_{b}+v_{c} i_{c}=L\left(\frac{d i_{a}}{d t} i_{a}+\right. \\
& \left.\frac{d i_{b}}{d t} i_{b}+\frac{d i_{c}}{d t} i_{c}\right)+v_{d c}\left(S_{a} i_{a}+S_{b} i_{b}+S_{c} i_{c}\right) \\
& q_{e s t}=\frac{1}{\sqrt{3}}\left(\left(v_{b}-v_{c}\right) i_{a}+\left(v_{c}-v_{a}\right) i_{b}+\right. \\
& \left.\left(v_{a}-v_{b}\right) i_{c}\right)=\frac{1}{\sqrt{3}}\left\{3 L\left(\frac{d i_{a}}{d t} i_{c}-\frac{d i_{c}}{d t} i_{a}\right)-\right. \\
& \left.v_{d c}\left[S_{a}\left(i_{b}-i_{c}\right)+S_{b}\left(i_{c}-i_{a}\right)+S_{c}\left(i_{a}-i_{b}\right)\right]\right\}
\end{aligned}
$$

As can be seen in Fig. 4, DC-link voltage is regulated by controlling $p$, and the desired power factor can be achieved setting $q$ reference. Differences between reference $\left(p^{*}, q^{*}\right)$ and estimated $\left(p_{\text {est }}, q_{\text {est }}\right)$ values of instantaneous powers are contrasted by means of two two-level hysteresis comparators, which provide two digital signals, $S p$ and $S q$, according to (11), where $H_{p}$ and $H_{q}$ represent hysteresis limit values. In any other cases not considered in (11), that is, those where respective instantaneous power value is located within hysteresis limits, comparators outputs stay at their previous value.

$$
\begin{aligned}
& S_{q}=1 \text { if } q_{e s t}-q^{*}<-H_{q} \\
& S_{q}=0 \text { if } q_{e s t}-q^{*}>+H_{q} \\
& S_{p}=1 \text { if } p_{e s t}-p^{*}<-H_{p} \\
& S_{p}=0 \text { if } p_{e s t}-p^{*}>+H_{p}
\end{aligned}
$$

Finally, as can be seen in Fig. 4, phase angle of estimated source voltages space vector (ESVSV) in $\alpha-\beta$ axes is converted to digital signal Sector, which indicates the position of that vector according to (12). This equation represents a division of $\alpha-\beta$ plane into 12 sectors, as shown in Fig. 5.

$$
(n-2) \frac{\pi}{6} \leq \theta_{n} \leq(n-1) \frac{\pi}{6} ; n=1,2 \ldots 12
$$

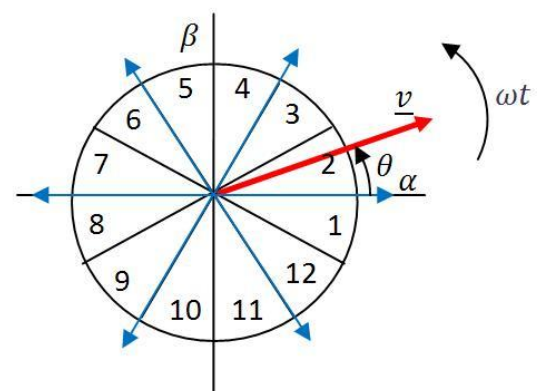

Fig. 5. Division of $\alpha-\beta$ plane into 12 sectors and estimated source voltages space vector

\section{Switching Table}

Most relevant element of DPC control strategy for threephase active rectifiers is the so-called Switching Table. Outputs from hysteresis controllers and ESVSV position are, in turn, inputs to the table, which stores, as a function of those inputs, the optimal commutation vectors for the rectifier to behave in a specific manner.

\section{A. Influence of commutation state vectors}

Each of the commutation state vectors may be sited in the $\alpha-\beta$ plane [5] according to the position where the space vector related to the converter AC-side phase voltages would be located in the case of applying the corresponding commutation state. This can be seen in Fig. 6.

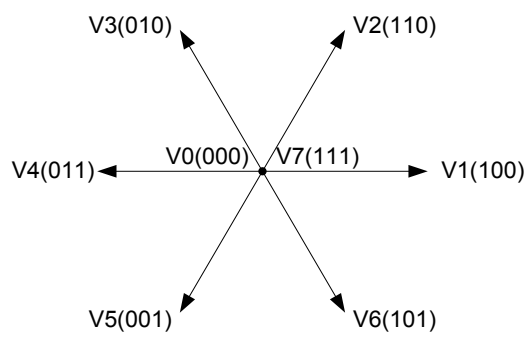

Fig. 6. Valid commutation state vectors

On the other hand and for each of the commutation state vectors, space vectors related to converter AC-side voltages present the $\alpha-\beta$ components shown in table II, where the corresponding converter AC-side phase voltages can also be observed [5]. 
TABLE II. - Converter AC-side voltages induced by commutation vectors

\begin{tabular}{|c|c|c|c|c|c|}
\hline Vector & $v_{a_{c o n v}}$ & $v_{b_{\text {conv }}}$ & $v_{c_{\text {conv }}}$ & $v_{c o n v_{\alpha}}$ & $v_{\operatorname{conv}_{\beta}}$ \\
\hline $\begin{array}{l}\text { V0 (000) } \\
\text { V7 (111) }\end{array}$ & 0 & 0 & 0 & 0 & 0 \\
\hline V1 (100) & $\frac{2}{3} v_{d c}$ & $-\frac{1}{3} v_{d c}$ & $-\frac{1}{3} v_{d c}$ & $\sqrt{\frac{2}{3}} v_{d c}$ & 0 \\
\hline V2 (110) & $\frac{1}{3} v_{d c}$ & $\frac{1}{3} v_{d c}$ & $-\frac{2}{3} v_{d c}$ & $\frac{1}{\sqrt{6}} v_{d c}$ & $\frac{1}{\sqrt{2}} v_{d c}$ \\
\hline V3 (010) & $-\frac{1}{3} v_{d c}$ & $\frac{2}{3} v_{d c}$ & $-\frac{1}{3} v_{d c}$ & $-\frac{1}{\sqrt{6}} v_{d c}$ & $\frac{1}{\sqrt{2}} v_{d c}$ \\
\hline V4 (011) & $-\frac{2}{3} v_{d c}$ & $\frac{1}{3} v_{d c}$ & $\frac{1}{3} v_{d c}$ & $-\sqrt{\frac{2}{3}} v_{d c}$ & 0 \\
\hline V5 (001) & $-\frac{1}{3} v_{d c}$ & $-\frac{1}{3} v_{d c}$ & $\frac{2}{3} v_{d c}$ & $-\frac{1}{\sqrt{6}} v_{d c}$ & $-\frac{1}{\sqrt{2}} v_{d c}$ \\
\hline V6 (101) & $\frac{1}{3} v_{d c}$ & $-\frac{2}{3} v_{d c}$ & $\frac{1}{3} v_{d c}$ & $\frac{1}{\sqrt{6}} v_{d c}$ & $-\frac{1}{\sqrt{2}} v_{d c}$ \\
\hline
\end{tabular}

\section{B. Switching Table design}

Equations for the input currents to an active three-phase rectifier can be expressed in $\alpha-\beta$ axes as seen in (13) [8].

$$
\begin{aligned}
& \frac{d i_{\alpha}}{d t}=\frac{1}{L}\left(v_{\alpha}-v_{c o n v_{\alpha}}-R i_{\alpha}\right) \\
& \frac{d i_{\beta}}{d t}=\frac{1}{L}\left(v_{\beta}-v_{c o n v_{\beta}}-R i_{\beta}\right)
\end{aligned}
$$

Combining equations (3) and (13), taking into account that $\theta=\omega t$ and neglecting resistor components, the previous equations can be obtained in d-q components as seen in (14).

$$
\begin{aligned}
& v_{d}=L \frac{d i_{d}}{d t}-\omega L i_{q}+v_{c o n v_{d}} \\
& v_{q}=L \frac{d i_{q}}{d t}+\omega L i_{d}+v_{c o n v_{q}}
\end{aligned}
$$

Equations representing instantaneous active and reactive powers in d-q reference system can be deduced from (3) and (5) and observed in (15).

$$
\begin{aligned}
& p=v_{d} i_{d}+v_{q} i_{q} \\
& q=v_{q} i_{d}-v_{d} i_{q}
\end{aligned}
$$

If ESVSV is made to coincide with d-axis in rotary $d-q$ system, its q-component would be null. On the other hand, as d-q system rotates at the same rate $(\omega)$ as the referred space vector does, time derivative of $v_{d}$ would also be null. Taking these facts and (14)-(15) into account, time derivatives of instantaneous active and reactive powers can be expressed as shown in (16) [8].

$$
\begin{aligned}
& \frac{d p}{d t}=v_{d} \frac{d i_{d}}{d t}=v_{d}\left(\frac{1}{L}\left(v_{d}-v_{c o n v_{d}}\right)+\omega i_{q}\right) \\
& \frac{d q}{d t}=-v_{d} \frac{d i_{q}}{d t}=-v_{d}\left(\frac{1}{L}\left(-v_{\text {conv }}\right)-\omega i_{d}\right)
\end{aligned}
$$

In connection with this, if $\omega i_{q}$ and $-\omega i_{d}$ terms are neglected and regarding ESVSV module $\left(|\underline{v}|=v_{d}=\right.$ $\sqrt{\frac{3}{2}} V_{\text {máx }}$, where $V_{\text {máx }}$ is the phase voltages amplitude), proportionality expressions (17) can be obtained [8].

$$
\begin{aligned}
& \frac{d p}{d t} \propto\left(\sqrt{\frac{3}{2}} V_{\text {máx }}-v_{c o n v_{d}}\right) \\
& \frac{d q}{d t} \propto\left(v_{\text {conv }_{q}}\right)
\end{aligned}
$$

It is easily deducible that $\mathrm{d}-\mathrm{q}$ components of converter AC-side voltages correspond to expressions (18).

$$
\begin{aligned}
v_{\text {conv }_{d}}= & \frac{1}{\sqrt{6}}\left(2 S_{a}-S_{b}-S_{c}\right) \cos (\omega t) \\
& +\frac{1}{\sqrt{2}}\left(S_{b}-S_{c}\right) \sin (\omega t) \\
v_{\text {conv }_{q}}= & \frac{1}{\sqrt{2}}\left(S_{b}-S_{c}\right) \cos (\omega t) \\
- & \frac{1}{\sqrt{6}}\left(2 S_{a}-S_{b}-S_{c}\right) \sin (\omega t)
\end{aligned}
$$

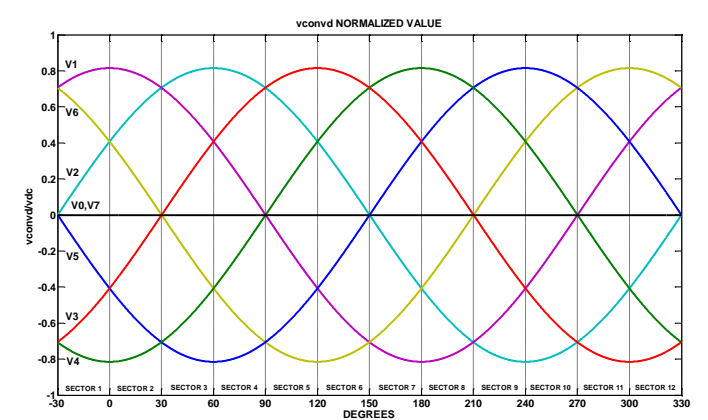

Fig. 7. Normalized value of $v_{c o n v_{d}}$ depending on sector and commutation vector

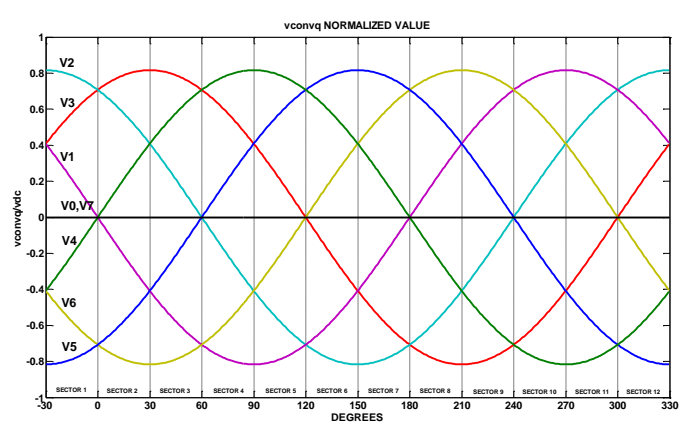

Fig. 8. Normalized value of $v_{\text {conv }}$ depending on sector and commutation vector 
Evolution of waveforms in (18) (normalized dividing by DC-link voltage, $v_{d c}$ ) can be plotted around $\alpha-\beta$ plane [8], which is divided into the 12 possible sectors where ESVSV can be located, for each of the eight possible commutation state vectors, as seen in Fig. 7 and Fig. 8. As can be seen, several valid commutation state vectors can be chosen within each sector in order to achieve a specific sign and magnitude for $v_{\text {conv }}$ and $v_{c o n v_{q}}$. Hence, a proper value for time derivatives of $p$ and $q$ can be obtained, as equation (17) demonstrates. Thus, it is possible to control instantaneous active and reactive powers and, therefore and respectively, converter DClink voltage and power factor. The most proper commutation vector, in each case, may be applied to the converter by previously storing them in a switching table, and, at all times, choosing the right one depending on the successive locations of ESVSV and the values of digital signals Sp and Sq.

\section{Implemented switching tables}

Two different switching tables have been used in this study. First of them is the one proposed in [8] (table ST1), which is designed trying to choose, at all times, the commutation state vector which leads to slower changes in instantaneous active and/or reactive powers (lower derivatives). In this way, the number of commutations within a specific time would decrease, as real instantaneous power values would need less rebounds between hysteresis limits within that time. Main problem of this approach lies in the smaller response rate of converter in the case of sudden high changes in references.

TABLE III. - Proposed switching table

\begin{tabular}{|c|c|c|c|c|c|c|c|c|c|c|c|c|c|}
\hline Sp & Sq & 1 & 2 & 3 & 4 & 5 & 6 & 7 & 8 & 9 & 10 & 11 & 12 \\
\hline \multirow{2}{*}{1} & 0 & V5 & V5 & V6 & V6 & V1 & V1 & V2 & V2 & V3 & V3 & V4 & V4 \\
\cline { 2 - 11 } & 1 & V3 & V4 & V4 & V5 & V5 & V6 & V6 & V1 & V1 & V2 & V2 & V3 \\
\hline \multirow{2}{*}{0} & 0 & V6 & V1 & V1 & V2 & V2 & V3 & V3 & V4 & V4 & V5 & V5 & V6 \\
\cline { 2 - 12 } & 1 & V1 & V2 & V2 & V3 & V3 & V4 & V4 & V5 & V5 & V6 & V6 & V1 \\
\hline
\end{tabular}

The second switching table (ST2) is a new one, proposed by the authors of this paper, and developed trying to achieve the opposite effect: fastest variations in instantaneous active and/or reactive power values (higher derivatives), which would induce contrary effects compared to those derived from table ST1. New table ST2 is shown in TABLE III, where Sp and Sq indicate whether the corresponding instantaneous power has to be increased (1) or decreased (0) (according to (11)), and selected commutation state vector is displayed as a function of $\mathrm{Sp}, \mathrm{Sq}$ and $\alpha-\beta$ plane sector where ESVSV is currently located.

\section{Model and simulation configurations}

Two Simulink models of a three-phase rectifier and its DPC system have been implemented regarding to the parameters that can be observed in Table IV. First one (Simple model) bases its control algorithm only on one of the mentioned switching tables, whereas the second one (Combined model) uses both of them. The Combined model works as follows: a secondary hysteresis band has been added to it, in such a way that, if the absolute value of the difference between one of the estimated instantaneous powers and its corresponding reference is beyond the limits marked by that band, ST2 would be utilized; otherwise, ST1 would be chosen. Thus, on the one hand, use of ST2 leads to sudden corrections in corresponding instantaneous power values when differences with respect to reference are relatively high (steps, for example), and, on the other hand, utilization of ST1 allows to achieve lower commutation frequencies in rectifier steady state, thanks to the slower changes that it involves.

In order to confirm these assumptions, a step in instantaneous active power reference has been introduced in both Simple and Combined models, and several measurements have been taken. So as to make these simulations possible, model in Fig. 4 is adapted in such a way that instantaneous active power reference is not achieved through DC-link control loop, but becomes the reference itself.

TABLE IV. - Model parameters

\begin{tabular}{|c|c|}
\hline Source voltage & $V_{L}=200 \mathrm{~V}$ \\
\hline Net impedance & $\begin{array}{c}R=0,012 \mathrm{~m} \Omega \\
L=0,2 \mu \mathrm{H}\end{array}$ \\
\hline $\begin{array}{c}\text { Connecting reactance } \\
\text { between net and converter }\end{array}$ & $\begin{array}{c}R=0,2 \mathrm{~m} \Omega \\
L=11 \mathrm{mH}\end{array}$ \\
\hline DC-link capacitor & $C=4,7 \mathrm{mF}$ \\
\hline Load resistor & $R_{L}=100 \Omega$ \\
\hline $\begin{array}{c}\text { Instantaneous reactive } \\
\text { power reference }\end{array}$ & $q^{*}=0 \mathrm{VAr}$ \\
\hline $\begin{array}{c}\text { Instantaneous active power } \\
\text { reference }\end{array}$ & $p^{*}=4000 \mathrm{VAr}$ \\
\hline Main hysteresis limits on $p$ & $H_{p}= \pm 80 \mathrm{~W}$ \\
\hline Main hysteresis limits on $q$ & $H_{q}= \pm 80 \mathrm{VAr}$ \\
\hline $\begin{array}{c}\text { Secondary hysteresis limits } \\
\text { on } p\end{array}$ & $H_{p}= \pm 150 \mathrm{~W}$ \\
\hline $\begin{array}{c}\text { Secondary hysteresis limits } \\
\text { on } q\end{array}$ & $H_{q}= \pm 150 \mathrm{VAr}$ \\
\hline
\end{tabular}

\section{Results and conclusions}

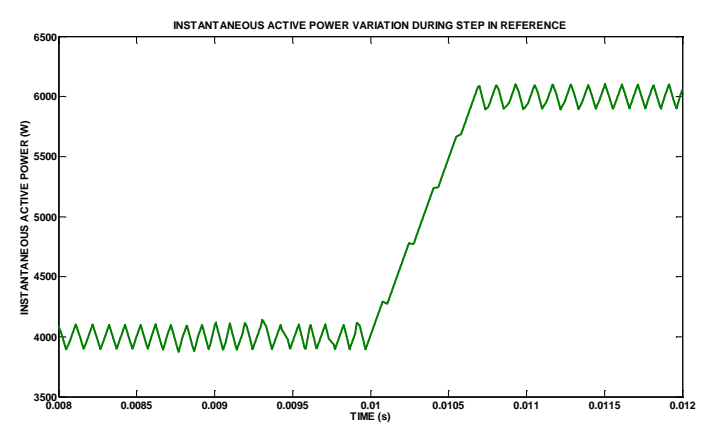

Fig.9. Effect of a step on real instantaneous active power in Simple model using ST1 


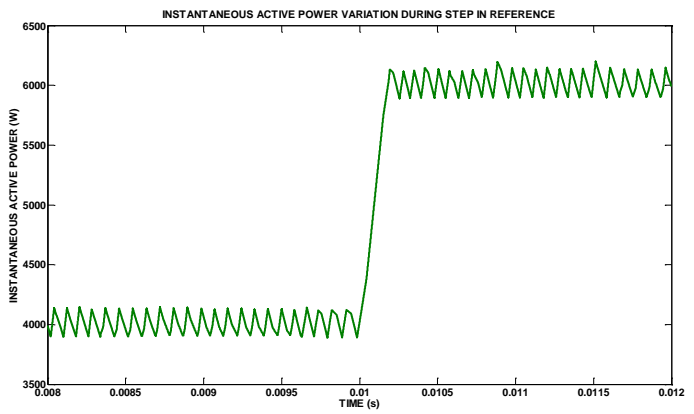

Fig.10. Effect of a step on real instantaneous active power in Simple model using ST2

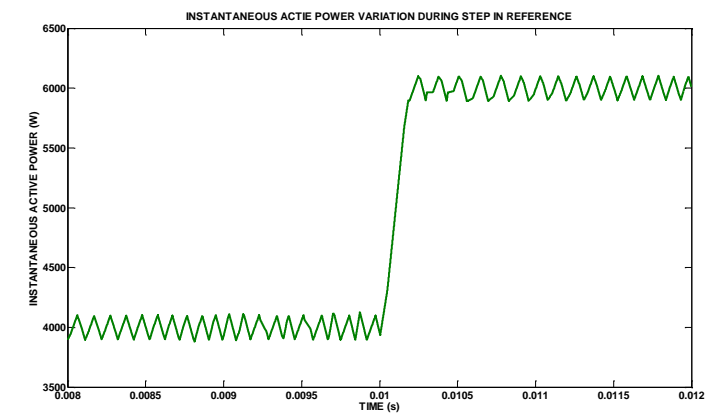

Fig. 11. Effect of a step on real instantaneous active power in Combined model

While Fig.9 and Fig.10 show the effect of a step on real instantaneous active power in the Simple model when using ST1 and ST2, respectively, Fig. 11 captures this effect on the Combined model.

In order to quantify the results visible to the naked eye in Fig. 9, Fig. 10 and Fig. 11, two measurements have been taken. On the one hand, Table $\mathrm{V}$ shows the number of commutations that occur during a $10 \mathrm{~ms}$ interval including the step. On the other hand, it can be seen in Table VI how long it takes the steady state to be recovered after the step.

TABLE V. - Number of commutations according to the implemented models

\begin{tabular}{|c|c|}
\hline Model & Number of commutations \\
\hline Simple model (ST1) & 161 \\
\hline Simple model (ST2) & 221 \\
\hline Combined model & 158 \\
\hline
\end{tabular}

TABLE VI. - Recovering time according to the implemented models

\begin{tabular}{|c|c|}
\hline Model & Time (ms) \\
\hline Simple model (ST1) & 0.7 \\
\hline Simple model (ST2) & 0.2 \\
\hline Combined model & 0.2 \\
\hline
\end{tabular}

As can be seen in tables V and VI, Combined model satisfies the advantages that separately characterize simple models. Therefore, the present approach concludes that Combined model may be more appropriate than simple models, as it leads to better dynamics and lower switching stress in three-phase active rectifiers.

\section{Acknowledgement}

The works that have led to the publication of this paper have been financed by the Spanish Science and Technology Ministry through the National Plan of Scientific Research, Development and Technological Innovation $(\mathrm{I}+\mathrm{D}+\mathrm{i})$ by means of the project with reference ENE2007-68032-C04-03/CON.

\section{References}

[1] M. P. Kazmierkowski and L. Malesani, "Current control techniques for three-phase voltage-source PWM converters: A survey," IEEE Trans. Ind. Electron., vol. 45, no. 5, pp. 691-703, Oct. 1998.

[2] D. Zhi, L. Xu, and B. W. Williams, "Improved direct power control of grid-connected DC/AC converters," IEEE Trans. Power Electronics, vol. 24, no. 5, pp. 1280-1292, May 2009.

[3] T. Noguchi, H. Tomiki, S. Kondo and I. Takahashi, "Direct power control of PWM converter without power-source voltage sensors", IEEE Trans. Ind. Applicat., May/June 1998 - Vol. 34, No.3, pp. 473 479.

[4] T. Ohnishi, "Three-phase PWM converter/inverter by means of instantaneous active and reactive power control", in Proc. IEEE IECON'91, pp. 819-824.

[5] S. Seguí, R. Masot, S. Orts and F. J. Gimeno, "Control vectorial de inversores trifásicos/1," Revista española de electrónica, no. 582, pp. 66-72, 2003.

[6] H. Akagi, E.H. Watanabe and M. Aredes, "Instantaneous power theory and applications to power conditioning”, Wiley-IEEE Press, 2007.

[7] P. Lezana and P. Cortés, "Control de un rectificador de frente activo trifásico," Seminario Avanzado de Electrónica Industrial, Universidad Técnica Federico Santa María, 2009.

[8] A. Baktash, A. Vahedi and M. A. S. Masoum, "Improved switching table for direct power control of three-phase PWM rectifier", in Australasian Universities Power Engineering Conference, 2007, pp. 1-5. 